\title{
Nucleosynthesis in the Outflow from Gamma Ray Burst Accretion Disks
}

\author{
R. Surman ${ }^{1}$, G. C. McLaughlin ${ }^{2}$ and W. R. Hix ${ }^{3}$
}

\begin{abstract}
We examine the nucleosynthesis products that are produced in the outflow from rapidly accreting disks. We find that the type of element synthesis varies dramatically with the degree of neutrino trapping in the disk and therefore the accretion rate of the disk. Disks with relatively high accretion rates such as $\dot{M}=10 M_{\odot} /$ s can produce very neutron rich nuclei that are found in the $r$ process. Disks with more moderate accretion rates can produce copious amounts of Nickel as well as the light elements such as Lithium and Boron. Disks with lower accretion rates such as $\dot{M}=1 M_{\odot} /$ s produce large amounts of Nickel as well as some unusual nuclei such as ${ }^{49} \mathrm{Ti}$, ${ }^{45} \mathrm{Sc},{ }^{64} \mathrm{Zn}$ and ${ }^{92} \mathrm{Mo}$. This wide array of potential nucleosynthesis products is due to the varying influence of electron neutrinos and antineutrinos emitted from the disk on the neutron-to-proton ratio in the outflow. We use a parameterization for the outflow and discuss our results in terms of entropy and outflow acceleration.
\end{abstract}

Subject headings: gamma ray:bursts-nucleosynthesis-accretion disks

\section{Introduction}

Data on long duration gamma ray bursts taken over the last few years has sparked the idea that these events are associated with core collapse supernovae, for a review see Mészáros (2002). Theoretical models, e.g. the collapsar model (Woosley 1993; MacFadyen \& Woosley 1999), suggest that a massive star collapses to an accretion disk surrounding a black hole, and a jet is driven out of the center of the star, leaving behind an escape route for the burst itself. Such an event will produce nucleosynthesis, not just in the the jet, but in the slower outflow that comes off of the accretion disk, as well as through explosive burning.

It is not known whether there are two types of events, core collapses that make "normal" supernovae with proto-neutron star cores and those that have accretion disks surrounding black holes

\footnotetext{
${ }^{1}$ Department of Physics and Astronomy, Union College, Schenectady, NY 12308

${ }^{2}$ Department of Physics, North Carolina State University, Raleigh, NC 27695-8202

${ }^{3}$ Physics Division, Oak Ridge National Laboratory, Oak Ridge, TN 37831-6374
}

that produce gamma ray bursts, or whether there is there a continuum of objects in between these extremes. The answer to the question, while of interest in its own right, is of crucial importance as far as our understanding of the significance of these objects for element synthesis. Estimates of the rate of gamma ray bursts from optical observations show that they occur on a timescale of less than about $10^{-2}$ times the rate of core collapse supernovae (Podsiadlowski et al 2004), while radio observations constrain the rate to be less than $5 \%$ of the Type $\mathrm{Ib} / \mathrm{c}$ rate (Berger et al 2003). However, accretion disks which form from stellar collapse may be a more common phenomenon, and and consequently element synthesis in outflow from an accretion disk may be much more frequent.

We have less observation evidence for the origin of short duration gamma ray bursts. Only one afterglow of a short (30 ms) gamma-ray burst has been detected thus far (GRB 050509b). Theoretical calculations have shown that these events may plausibly occur as a result of neutron star mergers, e.g. Ruffert \& Janka (1999, 2001); Rosswog \& Liebendoerfer (2003); Rosswog et al (2003). 
Numerical simulations of these events have produced very rapidly accreting disks which surround black holes with rates between $\dot{M}=1 M_{\odot} / \mathrm{s}$ and $\dot{M}=10 M_{\odot} / \mathrm{s}$.

We present a study of the nucleosynthesis products produced from the outflow from accretion disks of $\dot{M}=0.1 M_{\odot} / \mathrm{s}, \dot{M}=1 M_{\odot} / \mathrm{s}$, and $\dot{M}=10 M_{\odot} / \mathrm{s}$ which have black hole spin parameters of $a=0$. In general neutrino trapping, as a consequence of higher temperatures, will occur for lower accretion rate disks if the spin parameter is larger. We show an example of the element synthesis from an $a=0.95, \dot{M}=0.1 M_{\odot} /$ s disk to illustrate the effect of higher spin. Nucleosynthesis in gamma ray burst disk winds has also been studied by Pruet, Woosley, \& Hoffman (2003); Fujimoto et al. (2003, 2004).

Neutrinos can become trapped in the disks, particularly those that are associated with neutron star mergers. The emerging neutrinos annihilate and may drive the burst itself. Therefore neutrino trapping in these disks is under study by several groups, e.g. Lee et al (2004); Rosswog et al (2003); Kohri et al (2005); Janiuk et al (2005); Setiawan et al. (2004).

The neutrinos have an enormous influence on the final elemental abundances, even in cases where the neutrinos are not trapped in the disk. The disks are hot enough to release copious numbers of electron neutrinos and antineutrinos that then interact with free neutrons and protons in the outflow from the disk. Their influence over the neutron-to-proton ratio, together with entropy, and therefore heating of the material which leaves the disk, determines the final nucleosynthesis products.

We pay particular attention to the abundance of Nickel-56. Nickel-56 drives the light curves of core collapse supernovae. Since supernova light curves have been detected in gamma ray burst light curves, this implies substantial synthesis of Nickel-56 either in the outflow from the disk (Pruet, Thompson, \& Hoffman 2004) or in explosive burning (Maeda \& Nomoto 2003). The amount of Nickel observed in the light curves corresponds to roughly half of a solar mass (Woosley and Heger 2003).

We also examine some nuclei whose origin is poorly understood, but have large overproduc- tion factors in the outflows from low accretion rate disks, such as Titanium-44, Scandium- 45 and Zinc-64 (Pruet, Surman, \& McLaughlin 2004). We look at the light $p$-process nuclei such as ${ }^{92} \mathrm{Mo}$ and ${ }^{94}$ Mo. Rapidly accreting disks have been suggested as a site for the production of such light p-nuclei in Fujimoto et al. (2003).

We examine the prospects for producing an $r$ process in high accretion rate disks by way of material which is driven neutron rich by neutrino interactions as discussed in McLaughlin \& Surman (2005). And we also discuss conditions under which small amounts of very light nuclei such as Lithium and Boron can be produced in medium accretion rate disks.

\section{Disk and Outflow models}

We use disk models from Popham, Woosley, \& Fryer (1999) (PWF) for the low accretion rate disk, and from DiMatteo, Perna, and Narayan (2002) (DPN) for the high accretion rate disk. The PWF models do not include neutrino trapping and indeed for accretion rates less than $\dot{M}=0.1 M_{\odot} / \mathrm{s}$ there is very little trapping. For higher accretion rates neutrinos do become trapped and therefore we use the DPN models which include effects of neutrino trapping.

For the outflow we use the trajectories calculated in Surman \& McLaughlin (2005) and parameterize the outflow as a wind using

$$
|u|=v_{\infty}\left(1-\frac{R_{0}}{R}\right)^{\beta}
$$

where the starting position of the material is $R_{0}$ and $\beta$ determines the acceleration of the wind. Large beta represents slow acceleration whereas small beta represents rapid acceleration.

In the disk the entropy in units of Boltzmann's constant is $s / k \sim 10$. Since the material may be heated as it leaves the disk, we consider a range of entropies between $s / k=10$ and $s / k=50$.

We use the neutrino fluxes calculated in Surman \& McLaughlin (2004) for each of the the disk models we use here. In order to calculate the neutron-to-proton ratio in the outflowing trajectory we integrate of the flux coming from all parts of the disk, as described in Surman \& McLaughlin (2004). 
The place where the outflow occurs from the disk will have an impact on the nucleosynthesis, primarily because the neutrino flux varies over the length of the disk. In our calculations we use starting points of $r_{0}=100 \mathrm{~km}$ and $r_{0}=250 \mathrm{~km}$ from the black hole.

We begin the elemental evolution in the disk outflow by dynamically following the evolution of the electron fraction in the outflow, as described in Surman \& McLaughlin (2005). As the temperature drops below $10^{10} \mathrm{~K}$, we follow nuclear recombination using an intermediate network calculation (Hix \& Thielemann 1999) that includes strong, electromagnetic, and weak interactions. Finally we use a network that does solely neutron capture, photo-disintegration, beta decay, charged-current neutrino interactions, and betadelayed neutron emission (Surman \& Engel 2001). The latter is necessary for calculations of the most neutron rich elements.

\section{Nucleosynthesis from disks with Low Accretion Rates}

Using the outflow trajectories described in the previous section we present the results of reaction network calculations for outflow from a PWF disk with an accretion rate of $\dot{M}=0.1 M_{\odot} / \mathrm{s}$. This is representative of the accretion rates produced by the collapsar model (MacFadyen \& Woosley 1999).

In Fig 1 we show the nucleus with the largest mass fraction as a function of entropy and acceleration parameter. As can be seen from the figure, for high entropies Helium-4 has the largest mass fraction. The mass fractions of Helium-4 range from $X^{4} \mathrm{He}=0.30$ to $X^{4} \mathrm{He}=0.83$ in the region where it has a larger mass fraction than any other nucleus.

At high entropy there is a large number density of positrons which create protons in the outflow through the reaction $e^{+}+n \rightarrow p+\bar{\nu}_{e}$. At low entropies, there are less positrons since the material is electron degenerate, and therefore fewer protons. In addition for higher entropy, nuclear statistical equilibrium favors light nuclei until relatively low temperature. Thus the triple alpha reaction freezes-out of equilibrium before the majority of alpha particles can reassemble into heavy nuclei. For low entropies, iron peak nuclei assemble at high temperature, before the triple alpha reaction falls out of equilibrium.

For low entropies we find that nucleus with the largest mass fraction is ${ }^{62} \mathrm{Ni}$. The mass fraction in the region where ${ }^{62} \mathrm{Ni}$ dominates ranges from $X_{62} \mathrm{Ni}=0.25$ to $X_{62} \mathrm{Ni}=0.75$.

In the lower panel of Fig.1 we show the nucleus with the largest mass fraction excluding Helium-4. It is interesting to note that when large amounts of Helium-4 are produced, Nickel-56 is produced as well. For more rapid accelerations heavier isotopes are produced and for entropies of between $s / k=$ 30 and $s / k=40$ we see considerable Zinc- 66 .

Additional clues to the overall abundance pattern are given by the electron fraction, $Y_{e}=$ $1 /(1+n / p)$, where $n / p$ is the neutron to proton ratio. This is shown as the solid lines in Fig. 2. In addition to the effect of the entropy on the electron fraction as already discussed, there is a significant change due to the acceleration of the material. Slowly accelerating material (high $\beta$ ) has more time to approach the high equilibrium electron fraction (as set by the entropy) than the rapidly accelerating material, which retains some of the neutron richness of the disk. In addition, with fast acceleration (i.e. low $\beta$ ) there is little time for the neutrinos flowing off of the disk to interact with the outflow material. With slow acceleration, however, there is more time and the electron fraction increases.

In this relatively low accretion rate disk the neutrinos are not trapped, and therefore the neutrinos emitted from the disk are coming directly from an inverse beta decay. Since the disk is at low entropy, electrons in the disk dominate over positrons. Therefore more electron neutrinos are produced by inverse beta decay interactions than electron antineutrinos. The electron neutrinos interact with the outflowing matter to raise the electron fraction through the reaction $\nu_{e}+n \rightarrow p+e^{-}$. The effect of the neutrinos on the electron fraction here is relatively small, of the order of $1 \%-5 \%$; however, this influence grows significantly in trajectories from disks with higher black hole spin parameter $a$ and higher accretion rates.

In Fig. 2 the Nickel-56 abundance is shown. The largest abundance comes from moderate entropies and relatively slow outflows. Pruet, Surman, \& McLaughlin (2004) suggested that for these trajectories which produce consider- 
able Nickel-56 an unusual nucleosynthesis pattern is produced as well, including traditionally underproduced nuclei such as 45-Scandium, 49Titanium, and 64-Zinc. Here we extend the results of that study to include a larger range of trajectories that also include the effects of neutrino scattering. The results are shown in Fig. 3, which is a contour plot of the overproduction factors for these nuclei, where we define overproduction factor as in (Pruet, Surman, \& McLaughlin 2004)

$$
O(j)=\frac{M_{\text {wind }}}{M_{\text {SNejecta }}} \times \frac{X_{\text {wind }}}{X_{\text {solar }}},
$$

where $X_{\text {solar }}$ is the observed solar mass fraction of the nucleus, $X_{\text {wind }}$ is our calculated mass fraction in the outflow, $M_{S N e j e c t a}$ is the mass ejected in a supernova explosion, and $M_{\text {wind }}$ is the total mass contained in the outflow from the accretion disk. Here we take $M_{S N \text { ejecta }}$ to be $10 M_{\odot}$ and $M_{\text {wind }}$ to be $1 M_{\odot}$. All three of these nuclei have large overproduction factors that extend in a band from low entropy, slow acceleration to high entropy fast acceleration. The results correlate with electron fraction.

In Fig. 4 and 5 we consider also the light $p$ process nuclei, for which there is no universally agreed upon production site. It has been suggested in Fujimoto et al. (2003) that these nuclei can be produced in an accretion disk. We show Molybdinum-92 and Molybdinum-94 as well as Strontium-84, Krypton-78 and Selenium-74 in the context of the outflow from the low accretion rate disks. We find overproduction factors for ${ }^{92}$ Mo of greater than 1000 along the same band that overproduces large amounts of ${ }^{45} \mathrm{Sc},{ }^{49} \mathrm{Ti}$ and ${ }^{64} \mathrm{Zn}$.

The largest overproduction factors for Zinc occur for electron fractions at just slightly less than one half. This is similar to the alpha rich freezeout of the neutrino driven wind of the supernova (Fuller \& Meyer 1995; Hoffman et al 1996), but the overproductions we observe are larger. Overproduction factors greater than 10 occur for conditions that are just slightly proton rich, similar to what is observed in the hot bubble of the supernova (Pruet et al 2005; Frohlich et al. 2005). There is another band of large overproduction factor at slow outflow, which is due to the influence of neutrino capture on nucleons. It is interesting to note that in the observations of Cayrel et al.
(2004) the ratios $[\mathrm{Zn} / \mathrm{Fe}]$ and $[\mathrm{Zn} / \mathrm{Mg}]$ increase with decreasing metallicity. While the origin of Zinc is a matter of debate, Magnesium is thought to be primarily produced in core collapse supernovae.

Other nuclei that have large overproduction factors in these disks include ${ }^{46} \mathrm{Ti},{ }^{59} \mathrm{Co},{ }^{88} \mathrm{Sr},{ }^{90} \mathrm{Zr}$, ${ }^{74} \mathrm{Se}$ and ${ }^{63} \mathrm{Ni}$.

As mentioned above the black hole spin parameter increases the degree of trapping in the disk. In Fig. 6 we show the nickel-56 abundance for a PWF disk of $\dot{M}=1 M_{\odot} / \mathrm{s}$ and $a=0.95$. When compared with the electron fractions of the $a=0$ model shown in Fig. 2, it can be seen that the trapped neutrinos significantly change the neutron-to-proton ratio.

\section{Element Synthesis from Medium Ac- cretion Rate Disks}

Disks with accretion rates of $\dot{M}=1 M_{\odot} / \mathrm{s}$ are predicted by simulations of neutron star merger events. For these accretion disks the electron neutrinos are trapped in the disk. The antineutrinos are trapped as well, but in a much smaller region than for the neutrinos. The number of electron neutrinos overwhelms that of the electron antineutrinos coming from the disk although the antineutrinos have higher temperature than the neutrinos. For the DPN model, the neutrino temperatures range from 4.1 to $5.2 \mathrm{MeV}$ and the antineutrino temperatures range from 5.0 to $5.4 \mathrm{MeV}$ (Surman \& McLaughlin 2004). Due to the overwhelming numbers of neutrinos, in the outflowing material, the reaction $\nu_{e}+n \rightarrow p+e^{-}$creates very proton rich matter.

For the lower accretion rate disks considered in the last section, even the one with non-zero black hole spin parameter, the neutrinos make a $\lesssim 30 \%$ difference in the final value of the electron fraction, but for the disk considered in this section the neutrinos dominate the determination of the electron fraction and create a proton rich environment in the disk outflow for trajectories with all accelerations and entropies.

In the upper panel of Fig. 7 we show the nucleus with the largest mass fraction for a range of accelerations and entropies. As can be seen from the figure, helium again dominates the mass fraction for larger entropies, with mass fractions of $\sim 0.3$ 
- 0.6. In the lower panel of Fig. 7 we show the nucleus with the largest mass fraction excluding helium; again large amounts of ${ }^{56} \mathrm{Ni}$ are produced as well, with $X_{56} \mathrm{Ni} \sim 0.2$ to $X_{56} \mathrm{Ni} \sim 0.7$ as shown in Fig. 8.

It can be seen from Fig. 8, which shows the electron fractions in the outflow that the material becomes very proton rich, with $Y_{e}>0.7$ for slow accelerations. Given such proton rich material it is interesting to investigate whether very light nuclei such as Lithium and Boron are formed as well in the disk outflow. Table 1 shows the abundances of these elements for a few sample trajectories. For comparison, observational values of ${ }^{7} \mathrm{Li} / \mathrm{H}$ at low metallicity are in the range from $1 \times 10^{-10}$ to $2 \times 10^{-10}$ (Ryan et al 2000). However, only a fraction of the baryons in the universe will pass through massive stars, so the subject bears further investigation with a galactic chemical evolution study.

It is generally thought that Boron-11, which has a solar mass fraction of $X_{\odot,{ }^{11} \mathrm{~B}} \approx 5 \times 10^{-10}$, may be produced by a combination of cosmic ray spallation and the neutrino process, with estimates of up to $30 \%$ for the latter contribution VangioniFlam et al. (1996). For a discussion of LiBeB from related site, hypernovae ejecta, see Fields et al (2002).

In Fig. 8 the mass fraction of ${ }^{56} \mathrm{Ni}$ is plotted. Most of the outflowing material becomes Nickel-56 for moderate entropies $s / k \sim 30$, moderate outflows $\beta \sim 1.5$ or points in between. Little Nickel is produced fast outflows and low entropies as well as for high entropies and slow outflows. Our study suggests that if collapsing stars form disks which accrete at a rate of $\dot{M}=1 M_{\odot} / s$ or more slowly with higher spin parameter, then this range of acceleration and entropy would be required to explain the nickel inferred from the GRB light curve bumps.

\section{Element Synthesis from High Accretion Rate Disks}

In Fig. 9 we show the nucleus, or group of nuclei with the largest mass fraction for all of the outflow trajectories from the high accretion rate disk model, $\dot{M}=10 M_{\odot} / \mathrm{s}$. Rapid neutron capture process nuclei are produced abundantly for almost all the trajectories considered, except for those with very rapid acceleration and high entropy. The material in these trajectories becomes very neutron rich because of the influence of the neutrinos.

For such high accretion rates the electron antineutrinos are trapped in a fairly large region, within a radius of $158 \mathrm{~km}$ from the black hole, although the electron neutrinos are trapped in a larger region within $r_{\text {disk }} \sim 240 \mathrm{~km}$ (Surman \& McLaughlin 2004). The temperature of the electron antineutrinos is therefore higher, $3.6<T_{\bar{\nu}_{e}}<$ $6.9 \mathrm{MeV}$ as compared with $2.4<T_{\nu}<5.9 \mathrm{MeV}$. The neutrinos coming from the surface of the disk interact with the outflowing matter. Because the antineutrinos are more energetic than the neutrinos, the material is driven neutron rich through the reaction $\bar{\nu}_{e}+p \rightarrow n+e^{+}$which has a larger flux averaged cross section than $\nu_{e}+n \rightarrow p+e^{-}$.

The degree of neutron richness depends on the neutrino fluence: the time integrated neutrino flux experienced by a mass element in the outflow. For more slowly accelerating outflows the antineutrinos have more time to interact creating more neutrons. Conversely, for more rapid accelerations, there is little time for neutrino interactions and the electron fraction is higher.

We note that for lower entropies, the material is electron degenerate with very few positrons, and therefore has a large neutron-to-proton ratio regardless of its interactions with neutrinos or antineutrinos. We have also included the alpha effect as discussed in Meyer, McLaughlin \& Fuller (1998).

Fig. 10 we show the peaks which are primarily formed in the rapid neutron capture process. We calculate the ratios between the abundances in each peak region as in Meyer \& Brown (1997)

$$
\begin{aligned}
& \frac{130}{80}=\frac{\sum_{A=125}^{A=135} Y_{A}}{\sum_{A=85}^{A=75} Y_{A}} \\
& \frac{195}{130}=\frac{\sum_{A=190}^{A=200} Y_{A}}{\sum_{A=135}^{A=125} Y_{A}}
\end{aligned}
$$

The unshaded region is where the ratio $130 / 80$ is less than 0.07 ; only the $A=80$ peak is formed for these trajectories. The ratio $130 / 80$ is greater than 0.07 and the ratio $195 / 130$ is less than 0.3 for trajectories in the next region, labeled $A=130$ as it is this peak that primarily forms. The darkest shaded region is where the ratio $195 / 130$ is 
TABLE 1

Light Element Synthesis in Outflows from $\dot{m}=1$ Disks

\begin{tabular}{cccccc}
\hline \hline $\mathrm{S}$ & $\beta$ & $Y_{e}$ & ${ }^{X} \mathrm{He}$ & ${ }^{7} \mathrm{Li} / \mathrm{H}$ & ${ }^{11} \mathrm{~B} / \mathrm{H}$ \\
\hline 10 & 2.5 & 0.560 & 0.102 & $2.2 \times 10^{-9}$ & $6.7 \times 10^{-10}$ \\
15 & 2.5 & 0.641 & 0.191 & $9.6 \times 10^{-10}$ & $1.6 \times 10^{-10}$ \\
30 & 2.5 & 0.767 & 0.314 & $1.6 \times 10^{-10}$ & $1.1 \times 10^{-11}$ \\
40 & 2.5 & 0.779 & 0.372 & $3.7 \times 10^{-11}$ & $2.0 \times 10^{-12}$ \\
\hline
\end{tabular}

greater than 0.3 and thus the heaviest elements are formed. In the intermediately shaded region, the ratio $195 / 130$ is close to but below 0.3 ; in these trajectories the $A=195$ peak has formed, but the relative peak heights are not in agreement with the observed solar $r$-process abundances. A robust $r$ process occurs in the slow acceleration trajectories. The abundance pattern for two trajectories, one with fast outflow and one with low entropy is shown in Fig. 11.

For high entropy, the second, $A=130, r$ process peak is formed without the $A=195$ peak from somewhat more quickly accelerating trajectories. And for very fast accelerations we find only the $A=80$ peak is formed. It is interesting to note that both meteoritic data Qian \& Wasserburg (1998) and observations of metal poor halo stars, e.g. Sneden et al (2003), suggest two $r$-process sites. One of these forms the nuclei above the second peak and one of these forms the nuclei below. The meteoritic data suggests that the third peak is associated with a relatively frequently occurring event, such as core collapse supernovae. Core collapse supernova have been studied extensively as $r$-process sites beginning with Meyer et al (1992); Woosley et al (1994), although at present there is no completely self-consistent model that produces these elements without invoking new particle physics. The meteoritic data also hint that the low mass $r$-process nuclei are associated with a lower frequency event, such as a neutron star merger (Argast et al 2004).

Since the abundance distribution of the high mass nuclei in low metallicity halo star tracks well the solar system abundance, such an event should operate early in the evolution of the universe. An explosion of a massive star, such as in the collapsar model, would also operate early in the evolution of the universe. However, this model presently predicts lower accretion rate disks than the ones from which we find $r$-process being produced. In order to use this model to produce $r$-process nuclei, the disk would need to pass through a stage where it is very rapidly accreting or has a high black hole spin parameter.

The solar system abundances of lower mass $r$ process nuclei do not track the abundance pattern measured in metal poor stars well. This suggests that the type of event that produces these nuclei does not necessarily operate early in the evolution of the universe or is at least less frequent. If the lower mass $r$-process nuclei were to come from disks associated with neutron star mergers, then the outflow acceleration would be fast $\beta \sim 1$ for most possible values of entropy in the outflow. In Fig. 12 we plot the abundance pattern for two trajectories with entropies $s / k=30$ and $s / k=45$, both with $\beta=1.0$.

\section{Conclusions}

We find that a large range of nucleosynthesis may occur in the outflow from gamma ray burst accretion disks. For disks of $\dot{M}=0.1 M_{\odot} / s$ to $\dot{M}=1 M_{\odot} / s$, most of the element synthesis makes Helium and Nickel-56 except for low entropy and/or slow acceleration trajectories.

There is a significant, diagonal region of parameter space extending from slow outflow, low entropy to fast outflow high entropy trajectories, where nuclei such as ${ }^{45} \mathrm{Sc},{ }^{49} \mathrm{Ti},{ }^{64} \mathrm{Zn},{ }^{92} \mathrm{Mo}$, and ${ }^{94}$ Mo are overproduced by factors of $100-1000$ in the low accretion rate models. These low accretion rates are typical of the collapsar model. Disk winds can significantly contribute to the galactic inventory of these nuclei if the rate of supernovae 
which produce disks is $>10^{-2}$ times that of the rate of core collapse supernovae. This rate can be considerably higher than the limits on GRBs of $\lesssim 10^{-2}$ since not all disks may produce bursts.

In the outflow from both of these disks significant Nickel-56 is produced, with mass fractions of up to 0.7. Low accretion rate disks require slow outflow and moderate entropy, $s / k \sim 20$, to synthesize considerable Nickel-56, while moderate accretion rate disks can synthesize considerable Nickel with faster outflow for similar entropies. Introducing a non-zero spin parameter to the disk creates more ${ }^{56} \mathrm{Ni}$ at lower entropies and outflow accelerations, due to the increased influence of the neutrinos.

The moderate accretion rate disks can also make the $N=50$ peak of the $r$ process for low entropy and fast outflow. The moderate accretion rate disks are predicted to come from neutron star mergers. Observational evidence is consistent with such an infrequently occurring event which synthesizes the $A=80$ peak.

For the high accretion rate disks the majority of the parameter space produces an $r$ process. The $A=195$ peak is synthesized only for slowly accelerating outflows, although the $A=130$ peak is made more robustly. In the very fast acceleration, high entropy trajectories we find only the first peak of the $r$ process.

Future studies of the hydrodynamics of the outflow from accretion disks surrounding black holes will determine which of these patterns of element synthesis occurs in nature.

We thank Jason Pruet for useful discussions. We thank the Institute for Nuclear Theory at the University of Washington for its Hospitality and the Department of Energy for partial support during the completion of this work. This work was partially supported by the Department of Energy under contract DE-FG02-02ER41216 (GCM) and the Research Corporation under contract CC5994 (RS).

\section{REFERENCES}

Argast, D, Samland, M., Thielemann, F.-K. and Qian, Y.-Z. (2004) A\&A416 997.

Cayrel et al., 2004, A\&A416, 1117.
DiMatteo, T., Perna, R., \& Narayan, R. 2002 ApJ, 579,706

Berger, E., Kulkarni, S. R., Frail, D. A., \& Soderberg, A. M. 2003, ApJ, 599, 408

Fields, B. D., Daigne, F., Casse, M \& VangionFlam 2002 ApJ, 581, 389

Frail, D., et al. 2001, ApJ, 562, L55

Frohlich, C., Hauser, P. Liebendoerfer, M., G. Martinez-Pinedo, Thielemann, F.-K., Bravo, E., Zinner, N. T., Hix, W. R., Langanke, K., and Mezzacappa, A. and Nomoto, K. 2004 ApJsubmitted.

Fujimoto, S. Hashimoto, M., Arai, K., \& Matsuba R. 2004 ApJ614 847

Fujimoto, S., Hashimoto, M., Koike, Arai, K., \& Matsuba, R. 2003 ApJ, 585, 418

Fuller, G. M \& Meyer, B. S. 1995 ApJ453 792.

Heger, A., Fryer, C. L., Woosley, S. E., Langer, N., \& Hartmann, D. H. 2003, ApJ, 591, 288

Hix, W. R. \& Thielemann, F.-K. 1999 J. Comp. App. Math. 109, 321

Hoffman, R. D. Woosley, S. E., Fuller, G. M. and Meyer, B. S. 1996 ApJ460 478.

Janiuk, A., Perna, R., Di Matteo, T. \& Czerny, B. 2005 MNRAS355, 950

Kohri, K., Narayan, R. \& Piran, T. 2005, preprint (astro-ph/0502470)

Lee, W., Ramirez-Ruiz, E. \& Page, D. 2004 ApJ, 608, L5

MacFadyen, A. I. \& Woosley, S. E. 1999, ApJ, 524, 262

Maeda, K. \& Nomoto, K. 2003, ApJ, 598, 1163

McLaughlin, G. C., Fuller, G. M and Wilson, J. 1996 ApJ, 472, 400

McLaughlin, G. C \& Surman, R. S. Nuclear Physics (2005) A758, 189

Mészáros, P. 2002, ARA\&A, 40, 137 
B. Meyer, G. J. Mathews, W. M. Howard, S. E. Woosley, and R. D. Hoffman, Astrophys. J. 399, 656 (1992)

Meyer, Bradley S. \& Brown, Jason S. 1997 ApJS, 112, 199

B. Meyer, G. C. McLaughlin \& G. M. Fuller 1999 Phys. Rev. C58, 3696 (1998)

Podsiadlowski, P., Mazzali, P. A., Nomoto, K., Lazzati, D., \& Cappellaro, E. (2004) ApJ, 607, L17

Popham, R., Woosley, S. E., \& Fryer, C. 1999, ApJ, 518, 356

Pruet, J., Surman, R., \& McLaughlin, G. C. 2004, ApJ, 602, L101

Pruet, J., Woosley, S. E., Buras, R. Janka, H.-T. and Hoffman, R. D. 2005 ApJ623 325.

Pruet, J., Woosley, S. E., \& Hoffman, R. D. 2003, ApJ, 586, 1254

Pruet, J., Thompson, T., \& Hoffman, R. D. 2004, ApJ, 606, 1006

Qian, Y.-Z. \& Wasserburg 1998 ApJ, 494, 285

Rosswog, S \& Liebendoerfer 2003 MNRAS342, 673

Rosswog, S. et al 2003, MNRAS, 345, 1077

Ruffert, M., \& Janka, H.-T., 1999, A\&A, 344, 573

Ruffert, M., \& Janka, H.-T., 2001, A\&A, 380, 544

Ryan, S. G., Beers, T. C., Olive, K. A., Fields, B. D. \& Norris, J. E. 2000 ApJ, 530, L57

Setiawan, S., Ruffert, M., \& Janka, H-Th. 2004 MNRAS, 352, 753

Sneden et al. 2003 ApJ, 591, 936

Surman, R. \& Engel, J 2001 Phys. Rev. C, 035801.

Surman, R. \& McLaughlin, G. C. 2004, ApJ, 603, 611

Surman, R. \& McLaughlin, G. C. 2005, ApJ, 618, 397 ApJ, 507, L45

Woosley, S. E. 1993, ApJ, 405, 273
Woosley, S. E., Hartmann, D, Hoffman, R. D. \& W. C. Haxton 1990 ApJ, 365, 272

S. Woosley, J. R. Wilson, G. J. Mathews, R. D. Hoffman, and B. S. Meyer, Astrophys. J. 433, 299 (1994)

Woosley, S. E. and Heger, A. 2003, preprint (astro$\mathrm{ph} / 0309165)$

Vangioni-Flam, E, Casse, M., Fields, B. D. \& Olive, K. 1996 ApJ, 468, 199

This 2-column preprint was prepared with the AAS IATEX macros v5.2. 


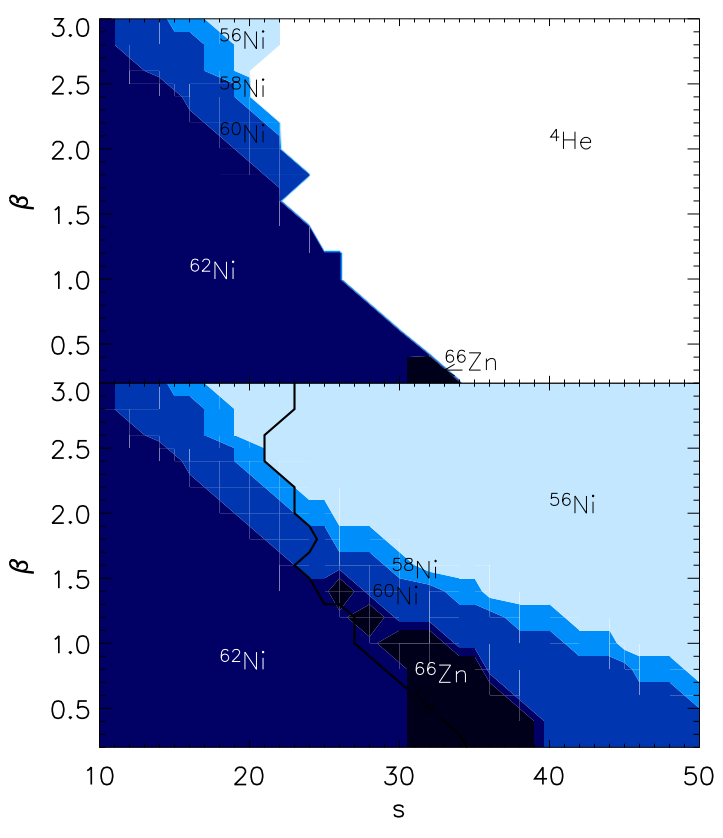

Fig. 1.- Shows the nuclei with the largest mass fraction in the outflow from a low accretion rate disk: $\dot{M}=0.1 \mathrm{M}_{\odot} /$ s. The vertical and horizontal axes are entropy per baryon, $s$, and the acceleration parameter, $\beta$. The lower panel shows the isotope with the largest mass fraction, excluding Helium-4.

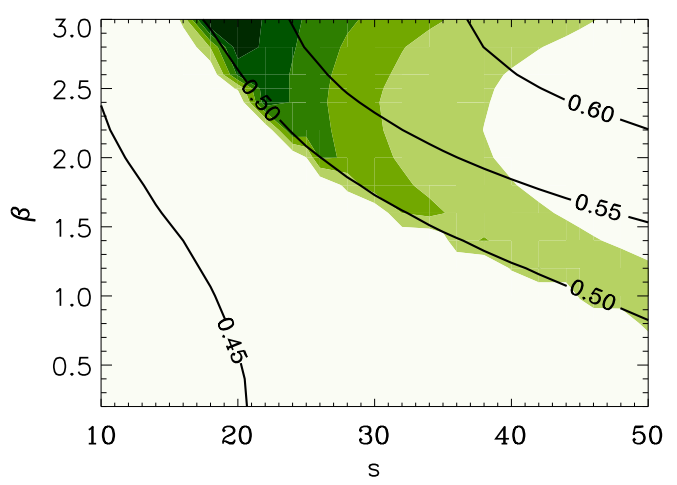

Fig. 2.- Shows the mass fraction of ${ }^{56} \mathrm{Ni}$ in the outflow of the low accretion rate disk. Dark shaded regions correspond, from darkest to lightest, to mass fractions of greater than 0.5, 0.4, 0.3, 0.2 , and 0.1 .

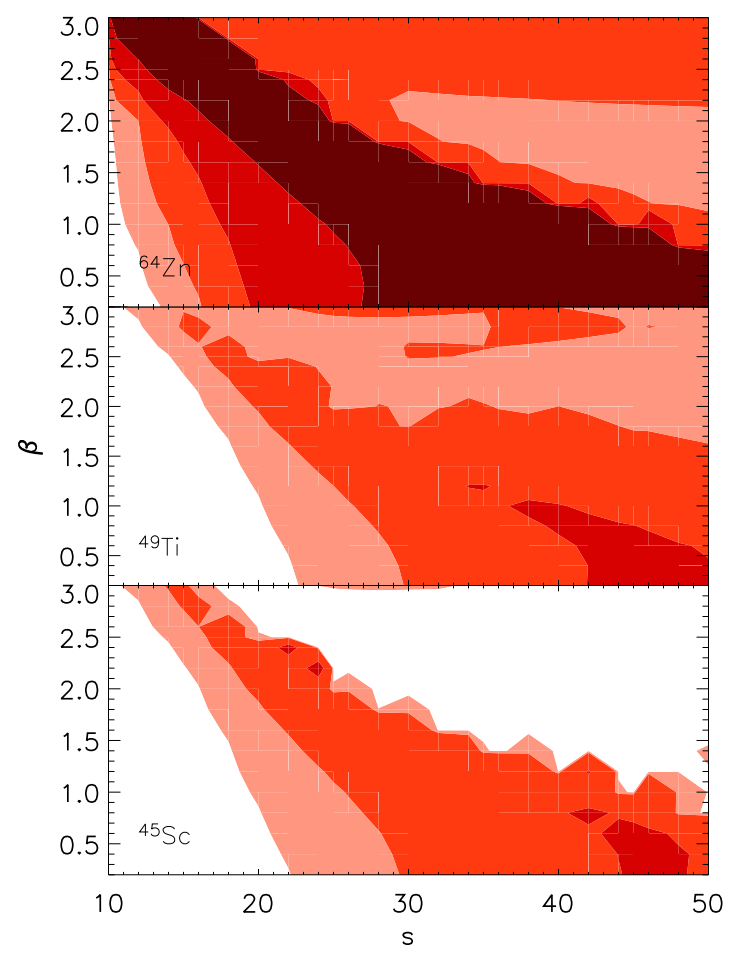

Fig. 3.- Shows the overproduction factor of ${ }^{64} \mathrm{Zn}$, ${ }^{49} \mathrm{Ti}$ and ${ }^{45} \mathrm{Sc}$, in the outflow of the low accretion rate disk. Dark shaded regions correspond to overproduction factors, from darkest to lightest, of greater than 1000, 100, 10, and 1. 


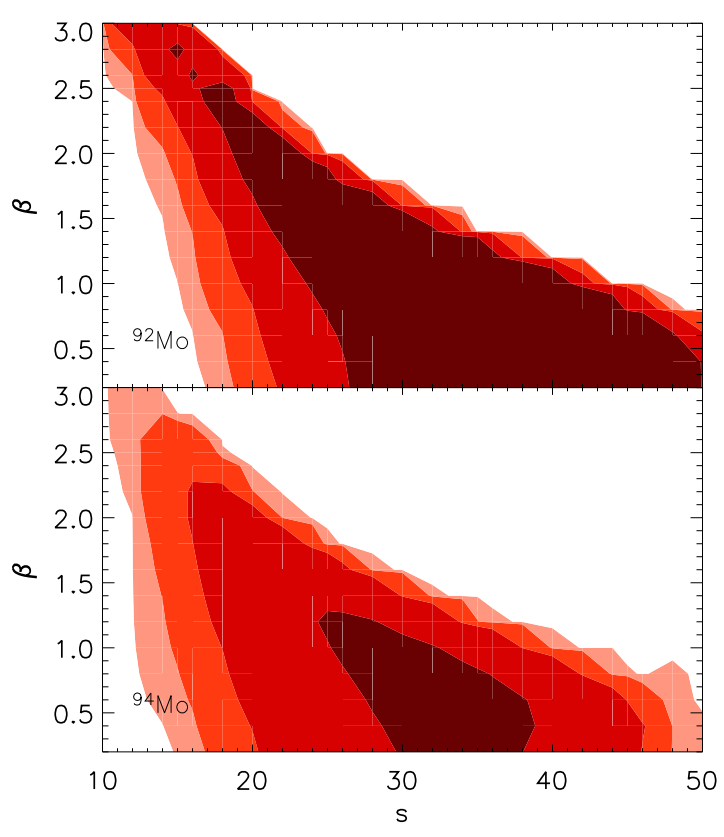

Fig. 4.- Shows the overproduction factor of ${ }^{92} \mathrm{Mo}$ and ${ }^{94} \mathrm{Mo}$ in the outflow of the low accretion rate disk. Dark shaded regions correspond to overproduction factors, in order from darkest to lightest of $1000,100,10,1$.

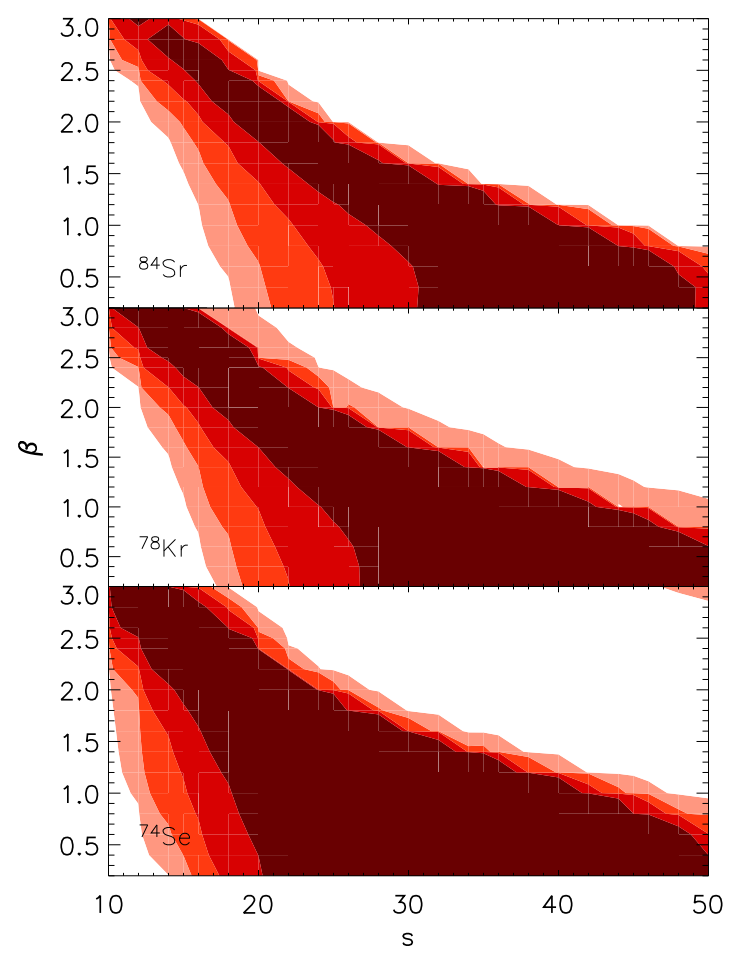

Fig. 5.- Shows the overproduction factors of the p-process nulcei ${ }^{84} \mathrm{Sr},{ }^{78} \mathrm{Kr}$, and ${ }^{74} \mathrm{Se}$ in the outflow of the low accretion rate disk. Dark shaded regions correspond to overproduction factors, in order from darkest to lightest of 1000, 100, 10, 1. 


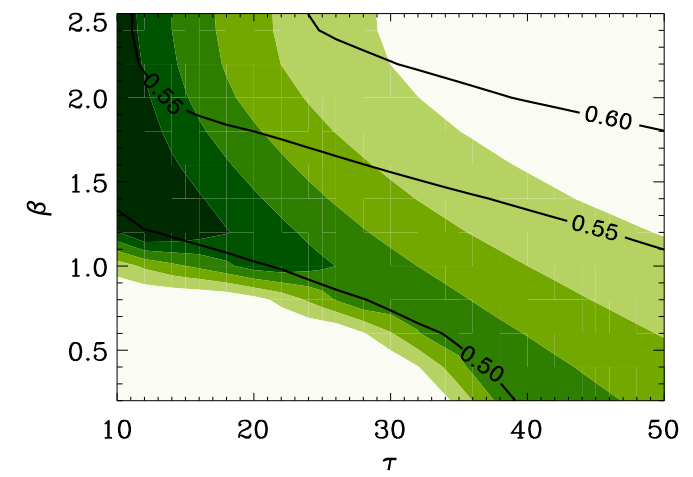

Fig. 6. - Shows the mass fraction of ${ }^{56} \mathrm{Ni}$ in the outflow of a low accretion rate disk, $\dot{M}=$ $0.1 \mathrm{M}_{\odot} / \mathrm{s}$, with black hole spin parameter $a=$ 0.95. Dark shaded regions correspond, from darkest to lightest, to mass fractions of greater than $0.5,0.4,0.3,0.2$, and 0.1 .

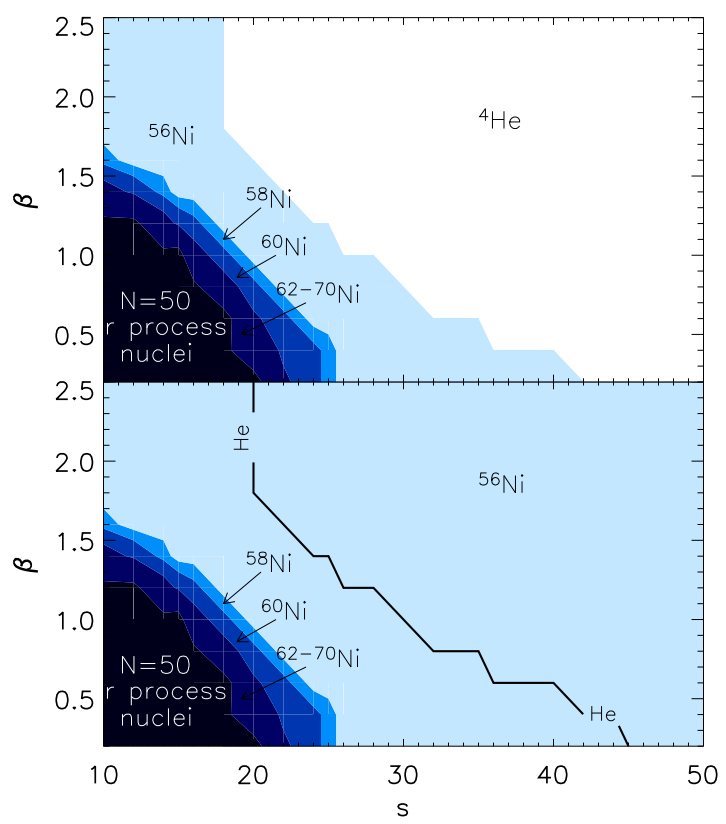

Fig. 7.- Shows the elements with the largest mass fractions for the medium accretion rate disk of $\dot{M}=1 M_{\odot} / \mathrm{s}$. The top panel shows the element with the largest mass fraction and the bottom panel shows the same excluding $\mathrm{He}$.

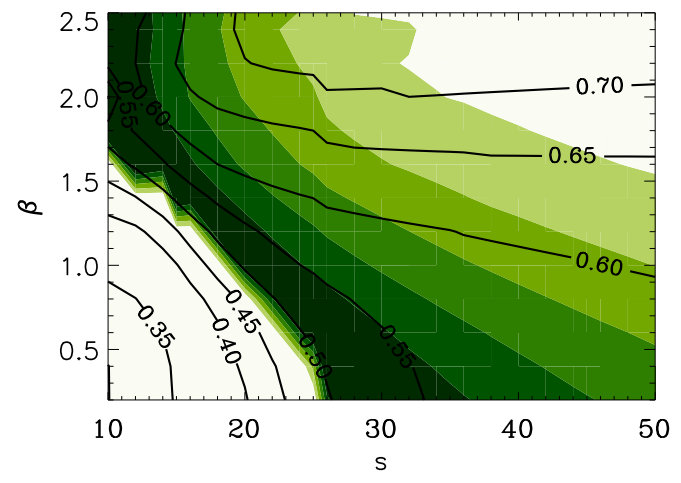

Fig. 8.- Shows the mass fraction of ${ }^{56} \mathrm{Ni}$ in the outflow of the low accretion rate disk. Dark shaded regions correspond, in order from darkest to lightest, to mass fractions of $0.5,0.4,0.3,0.2$, and 0.1 .

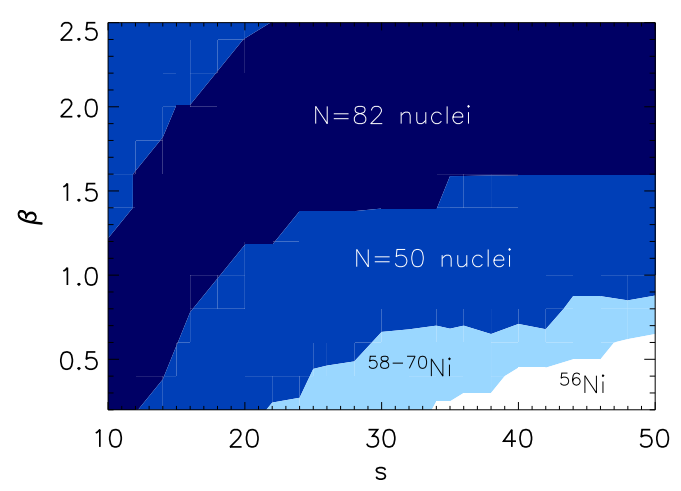

Fig. 9.- Shows the elements with the largest mass fractions for the high accretion rate disk, DPN $\dot{M}=10 M_{\odot} / \mathrm{s}$. 


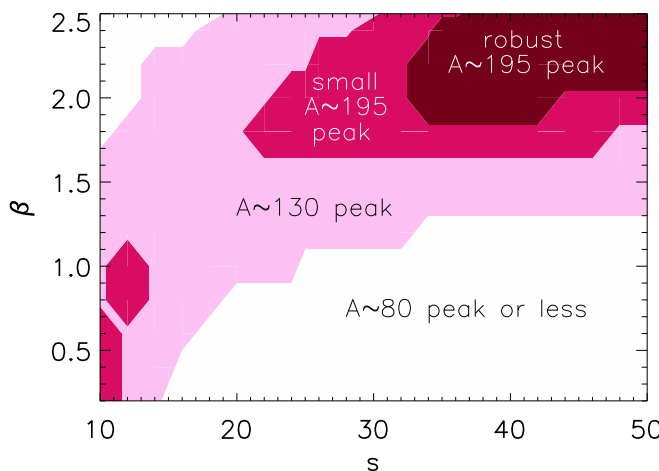

Fig. 10.- For the high accretion rate disk, $\dot{M}=$ $10 M_{\odot} / \mathrm{s}$, shows the region where the second and third peaks of the $r$ process form.

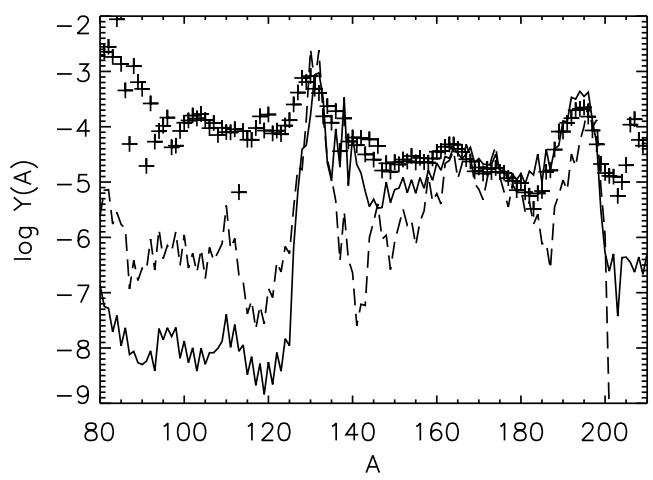

Fig. 11. - Shows a final abundance pattern for outflow from the high accretion rate disk with parameters $s / k=10, \beta=0.2$ (dashed line) and $s / k=50, \beta=2.2$ (solid line). Scaled solar system $r$-process abundances are shown for comparison (crosses).

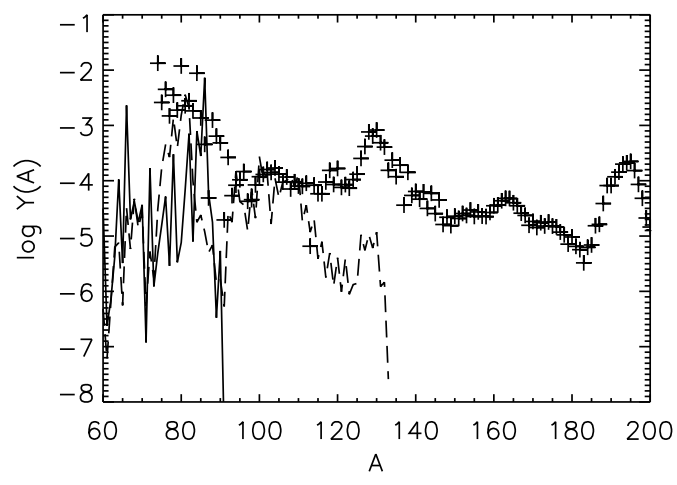

Fig. 12.- Shows a final abundance pattern for outflow from the high accretion rate disk with parameters $\beta=1.0$ and $s / k=30$ (dashed line) and $s / k=45$ (solid line). These trajectories give only the first peak of the $r$ process. 